\title{
Sub-electron Charge Relaxation via 2D Hopping Conductors
}

\author{
Yusuf A. Kinkhabwala†and Konstantin K. Likharev† \\ $\dagger$ Department of Physics and Astronomy, Stony Brook University, Stony Brook, NY \\ 11794-3800
}

\begin{abstract}
We have extended Monte Carlo simulations of hopping transport in completely disordered 2D conductors to the process of external charge relaxation. In this situation, a conductor of area $L \times W$ shunts an external capacitor $C$ with initial charge $Q_{i}$. At low temperatures, the charge relaxation process stops at some "residual" charge value corresponding to the effective threshold of the Coulomb blockade of hopping. We have calculated the r.m.s. value $Q_{R}$ of the residual charge for a statistical ensemble of capacitor-shunting conductors with random distribution of localized sites in space and energy and random $Q_{i}$, as a function of macroscopic parameters of the system. Rather unexpectedly, $Q_{R}$ has turned out to depend only on some parameter combination: $X_{0} \equiv L W \nu_{0} e^{2} / C$ for negligible Coulomb interaction and $X_{\chi} \equiv L W \kappa^{2} / C^{2}$ for substantial interaction. (Here $\nu_{0}$ is the seed density of localized states, while $\kappa$ is the dielectric constant.) For sufficiently large conductors, both functions $Q_{R} / e=F(X)$ follow the power law $F(X)=D X^{-\beta}$, but with different exponents: $\beta=0.41 \pm 0.01$ for negligible and $\beta=0.28 \pm 0.01$ for significant Coulomb interaction. We have been able to derive this law analytically for the former (most practical) case, and also explain the scaling (but not the exact value of the exponent) for the latter case. In conclusion, we discuss possible applications of the sub-electron charge transfer for "grounding" random background charge in single-electron devices.
\end{abstract}

PACS numbers: 72.20.Ee, 73.23.Hk, 73.40.RW 


\section{Introduction}

Electron transport via inelastic hops between localized states in disordered conductors has been studied for many years, with the main focus on the average transport characteristics (e.g., dc current dependence on temperature and applied electric field) and to a lesser extent on the $1 / f$ noise - see Refs. [1, 2, 3, 4, for comprehensive reviews of this work. The relatively recent observation [5, 6, 7] that hopping transport may provide quasi-continuous ("sub-electron") charge transfer gave a motivation for the extension of this work to the statistics of the electric charge $Q$ carried over by the hopping current.

The idea of the quasi-continuous charge transfer is quite simple: due to the electrostatic polarization, each electron hop between two localized sites inside the conductor leads to a step-like increase of the "external charge" $Q(t)$, which may be defined as the time integral of current $I(t)$ flowing through the wires connecting the conductor's electrodes to the electric field source. If an electron is transferred through the whole sample in one hop (as happens in the usual tunnel junctions), the charge step $|\Delta Q|$ is equal to the fundamental charge $e$. However, if an electron in an extended conductor hops between two sites which are separated by a distance $\Delta r$ much less than the conductor length $L$, then the step $|\Delta Q|$ is of the order of $e \times(|\Delta r| / L) \ll e$. (The exact expression depends on the sample and electrode geometry.) This means that the charge transport becomes nearly continuous, just as in long diffusive conductors [7, 8]. This phenomenon may have several useful applications in single-electronics, especially since the hopping conductors (in contrast to their diffusive counterparts) may provide the necessary high values of resistance $R \gg \hbar / e^{2}[9]$ without adding too much stray capacitance to that of single-electron islands.

One of the manifestations of the quasi-continuous charge transport is the suppression of the shot noise [4, 10, 11]. Namely, for sufficiently small values of the observation frequency $f$ (with a possible exception for the $1 / f$ noise at very low frequencies) the current noise spectral density $S_{I}(f)$ becomes approximately $L_{c} / L \ll 1$ times the Schottky value $2 e I$, where $L_{c}$ is some characteristic length scale. This prediction [5] has been confirmed in several recent experimental [12, 13] and theoretical [14, 15, 16, 17] studies of hopping.

The goal of this work has been to study another manifestation of the quasicontinuous charge transfer at hopping, which is more closely related to its most important potential application: the ability to "ground" sub-electron amounts of electric charge [9]. For this, we have analyzed the simple system shown in Fig. 1] a hopping conductor shunts an external capacitance $C$ with an initial charge $Q_{i}$. The capacitance charge $Q$ leads to a nonvanishing electric field $E=V / L=Q / C L$ applied to the conductor, which causes electrons to hop through the conductor. These hops result in the gradual reduction of the charge $Q$ and hence the field $E$. At the perfectly continuous ("Ohmic") conduction the process would continue until $Q$ and $E$ vanished completely (at $T \rightarrow 0$ ); however, for hopping conductors of a finite size $L \times W$ the charge relaxation stops at a certain finite residual charge which generally depends not only on 


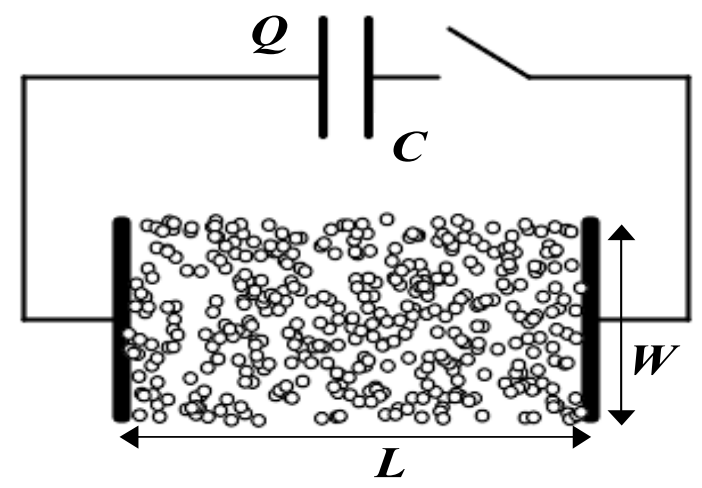

Figure 1. The system under analysis (schematically).

the macroscopic parameters of the system, but also on the particular distribution of the localized sites over space and energy and on the initial charge $Q_{i}$.

Though qualitative experimental evidence of sub-electron charge relaxation has been obtained long ago [18, 19], to the best of our knowledge this phenomenon has never been studied in detail. The objective of this work has been to study the dynamics of this charge relaxation process, and the statistics of the residual charge theoretically. The problem is essentially classical, but multi-particle, highly nonlinear, and statistical, so that most results have to be obtained by numerical (Monte Carlo) simulation using modern supercomputer facilities (see the Acknowledgments section below).

\section{Model}

For the hopping conductor, we have used the same model whose average transport characteristics and current noise had been extensively explored recently for the case of fixed, constant applied field $E$ [16, 17]. Briefly, the conductor is "fully frustrated" in the sense that the localized sites are randomly and uniformly distributed, with a constant "seed" density of states $\nu_{0}$, over both the rectangular $2 \mathrm{D}$ sample of area $L \times W$ and a broad interval of "seed" energies $\varepsilon^{(0)}$. The full energy $U$ of the system is the sum of the "seed" energies of all occupied sites and the electrostatic energy of the Coulomb interaction of the hopping electrons with each other and the external capacitance:

$$
U=\sum_{j} n_{j} \varepsilon_{j}^{(0)}+\frac{e^{2}}{2 \kappa} \sum_{j, k \neq j}\left(n_{j}-\frac{1}{2}\right)\left(n_{k}-\frac{1}{2}\right) G\left(\mathbf{r}_{j}, \mathbf{r}_{k}\right)+\frac{Q^{2}}{2 C} .
$$

Here $n_{j}$ (equal to either 0 or 1 ) is the occupation number of the $j^{\text {th }}$ localized site, while $\kappa$ is the dielectric constant of the insulating environment [20]. For the simplest geometry of a 2D conductor connecting two semi-space-shaped electrodes, the Green's function $G$ in Eq. (11) may be simply expressed as a sum over the infinite set of image charges in 
the electrodes:

$$
\begin{gathered}
G\left(\mathbf{r}_{j}, \mathbf{r}_{k}\right)=\sum_{n=-\infty}^{\infty}\left[\frac{1}{\sqrt{\left(2 n L+x_{k}-x_{j}\right)^{2}+\left(y_{k}-y_{j}\right)^{2}}}\right. \\
\left.-\frac{1}{\sqrt{\left(2 n L+x_{k}+x_{j}\right)^{2}+\left(y_{k}-y_{j}\right)^{2}}}\right] .
\end{gathered}
$$

For this geometrical model, the total charge $Q$ of the capacitor (including the polarization component) is

$$
Q=Q_{i}-\left[N_{e} e+\sum_{j} e\left(n_{j}-\frac{1}{2}\right) \frac{x_{j}}{L}\right],
$$

where $Q_{i}$ is the initial charge and $x_{j}$ is the $j^{\text {th }}$ site position along the sample length $L$, while $N_{e}$ is the total number of electrons that have passed through the conductor from the start of the relaxation process until the given moment. In the limit of large charge $\left(|Q| \gg Q_{R}\right)$ the effect of capacitance on hopping transport is equivalent to that of the electric field $E=Q / C L$.

Electron hops are permitted from any occupied site $j$ to any unoccupied site $k$ with the rate

$$
\gamma_{j k}=\Gamma_{j k} \exp \left(-\frac{r_{j k}}{a}\right)
$$

where $a$ is the localization length, and

$$
\hbar \Gamma_{j k}\left(\Delta U_{j k}\right)=g \frac{\Delta U_{j k}}{1-\exp \left(-\Delta U_{j k} / k_{B} T\right)} .
$$

Here $\Delta U_{j k}$ is the difference of the total system energy $U$ before and after the hop, and $g$ is a small dimensionless parameter which affects only the scale of hopping conductivity $\sigma_{0} \equiv g e^{2} / \hbar$. The numerical study has been carried out using the classical Monte Carlo technique by Bortz, Kalos and Leibowitz [21] in the form suggested by Bakhvalov et al. 22, which has become the de facto standard for simulations of single-electron tunneling [23. An important feature of this algorithm is that it is not slowed down by the gradual reduction of hopping rates at charge relaxation.

\section{Charge Relaxation Dynamics}

Figure 2 shows, by thin lines, typical results of our Monte Carlo simulations for two values of the dimensionless parameter of the Coulomb interaction strength, $\chi \equiv e^{2} \nu_{0} a / \kappa$. Note the logarithmic time scale and the linear scale of $Q$; in such coordinates the exponential relaxation of average charge in an $R C$ circuit with a linear Ohmic resistor looks like a sharp step down at $t \approx R C$. We indeed observe such behavior at hopping when the initial electric field is low, i.e. in the high temperature limit. However, motivated by prospects of practical applications [9], our main focus is on the opposite, "high-field" (low-temperature) limit. Figure 2 shows that in this case the dynamics of 
discharge through the hopping conductor is rather different: it slows down dramatically at $Q \rightarrow 0$. This is exactly what should be expected from the previous studies of variablerange hopping at constant applied field, which show that the hopping conductance drops exponentially as the field decreases [1, 2, 3, 15, 16, 17. A qualitatively similar dynamics is also typical for the qualitatively close (but quantitatively different) problem of intrinsic relaxation in electron glasses - see, e.g., recent publications [24, 25, 26] and prior work cited therein.

It has turned out that most of the relaxation process, while the charge is sufficiently large $\left(|Q| \gg Q_{R}\right)$, may be well described by the mean-field equation

$$
\frac{d Q}{d t}=-I(T, E, \chi)=-\sigma(T, E, \chi) E W
$$

where $\sigma(T, E, \chi)$ is the nonlinear conductance in the constant applied field $E$. In the low-temperature limit $\left(k_{B} T \ll e E r\right.$, where $r$ is the average length of the hops contributing substantially into the current), we can use the following analytical expressions obtained by fitting the results of our numerical simulations of constant-field hopping within the same model [16, 17]:

(i) If Coulomb interaction is negligible, $\chi^{3} \ll E / E_{0}$,

$$
\frac{\sigma}{\sigma_{0}} \approx A(E, 0) \exp \left[-\left(B(E, 0) \frac{E_{0}}{E}\right)^{1 / 3}\right],
$$

where $e E_{0} a \equiv 1 / \nu_{0} a^{2}$, while $A(E, \chi)$ and $B(E, \chi)$ are dimensionless, weak functions of the applied field $E$ and Coulomb interaction strength $\chi$. In a prior study [16], we have found the best fit for the pre-exponential (model-specific) function to be $A(E, 0)=$ $(9.2 \pm 0.6)\left(E / E_{0}\right)^{(0.80 \pm 0.02)}$, with $B$ treated as a constant: $B(E, 0)=0.65 \pm 0.02$.

(ii) If Coulomb effects are substantial, then

$$
\frac{\sigma}{\sigma_{0}} \approx A(E, \chi) \exp \left[-\left(B(E, \chi) \frac{\chi E_{0}}{E}\right)^{1 / 2}\right] .
$$

For the particular value of $\chi=0.5$, a similar approach to fitting gives [17] $A(E, 0.5)=$ $(3.0 \pm 0.4)\left(E / E_{0}\right)^{(0.72 \pm 0.07)}$ with $B(E, 0.5)=1.68 \pm 0.07$.

For relatively low fields, $E \ll E_{0}$, these formulas describe the so-called "high-field" variable range hopping [27, 28, 29, 30, 31].

Broad gray curves in Fig. 2 show the results of integration of the mean field equation using these formulas for one value of capacitance $C / C_{0}=100$. (The middle curves correspond to the best fit values, while the outer curves reflect the fitting uncertainties specified above.) One can see that at $|Q| \gg Q_{R}$ the relaxation results may be well described by the mean-field approach. However, this approach does not work at $Q \rightarrow 0$ where it predicts the complete relaxation of charge, while in reality (and numerical experiment) the process stalls at a certain "residual" charge. 

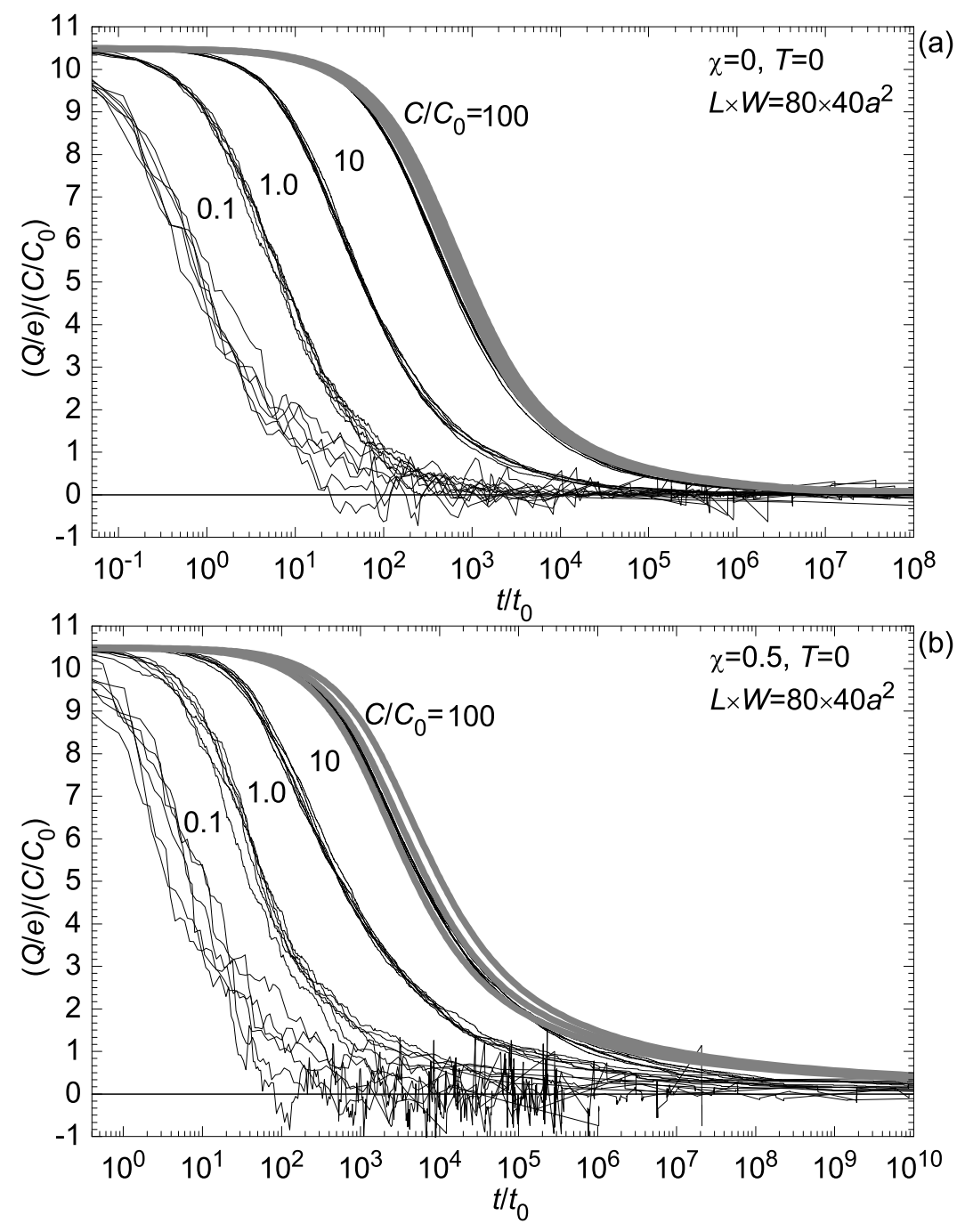

Figure 2. Capacitance charge $Q$ relaxation (at $T=0$ ) for the cases of (a) negligible $(\chi=0)$ and $(b)$ substantial $(\chi=0.5)$ Coulomb interaction of hopping electrons. Thin lines show Monte Carlo results (for 6 realizations of each case) for several values of external capacitance $C$, with fixed conductor size $L \times W=80 \times 40 a^{2}$. The thick gray curves correspond to the results of the solution of Eq. (6) with Eq. (7) for panel (a) and Eq. (8) for panel (b) for $C / C_{0}=100$, with the central curve corresponding to the best-fit parameters $A$ and $B$ and the outer curves corresponding to the uncertainty in these parameters. (See the text.) Time is measured in units of $t_{0} \equiv \hbar \nu_{0} a^{2} / g$, while capacitance is expressed in units of $C_{0} \equiv e^{2} \nu_{0} a^{2}$. 


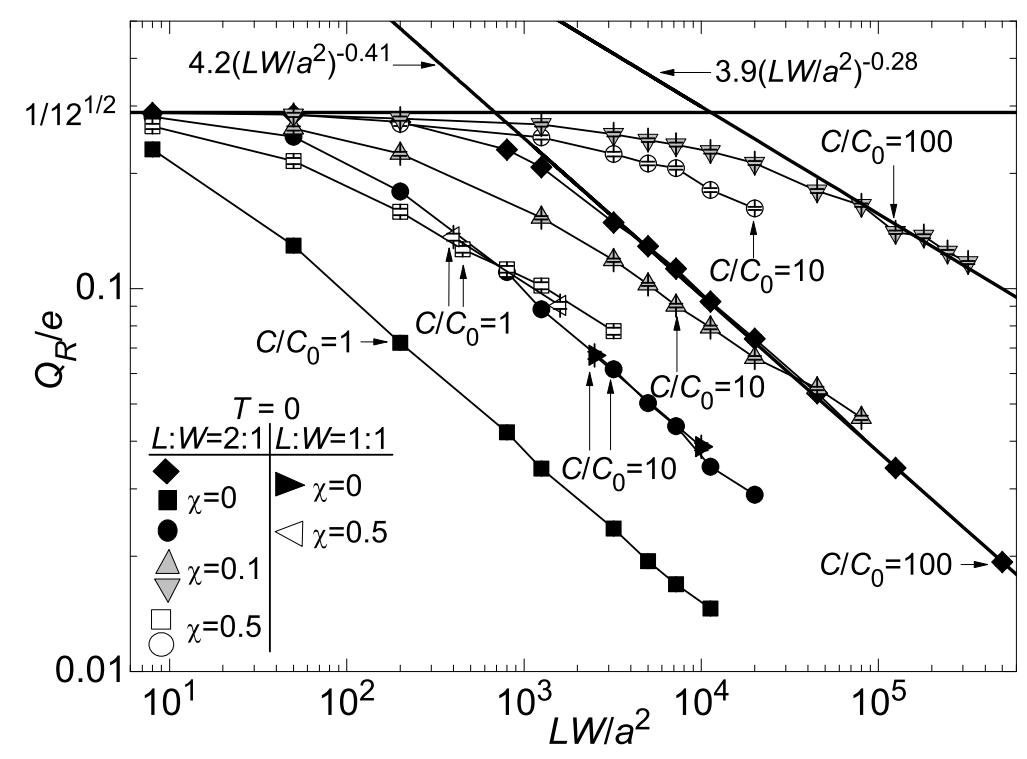

Figure 3. The r.m.s. value $Q_{R}$ of the residual charge at $T=0$ for negligible $(\chi=0)$ and finite $(\chi=0.1$ and 0.5$)$ Coulomb interaction, as a function of the conductor area $(L \times W)$ for different external capacitances $C$, and two different aspect ratios ( $L: W$ $=2: 1$ and 1:1). Each point represents data averaged over a large number $\left(10^{3}\right)$ of conductor samples with vertical error bars corresponding to the uncertainty of such averaging. (Error bars are shown on figure, unless smaller than the symbol size). Thin lines are only guides for the eye. The bold horizontal line corresponds to Eq. (9), while the bold tilted lines are the best power-law fits for large-sample data.

\section{Residual Charge Statistics}

Figure 3 shows some of our results for the r.m.s. value $Q_{R}$ of the residual charge, obtained for a broad range of "macroscopic" parameters of the system, including external capacitance $C$ and normalized Coulomb interaction strength $\chi$, as a function of the conductor area $L \times W$. (These results do not change noticeably if the systems are annealed after the relaxation.)

For sufficiently small samples, the number of localized sites is so low that no internal hopping events may occur within the energy interval of interest, and the initial charge can only relax by direct tunneling between the electrodes, giving changes of $Q$ in multiples of $e$. The Coulomb blockade theory (see, e.g., Ref. [5]) shows that at low temperatures such tunneling is blocked at $|Q|<e / 2$. If the initial charge $Q_{i}$ is random (as has been accepted in our calculations), then the residual charge is uniformly distributed within the range from $-e / 2$ to $+e / 2$, and the r.m.s. residual charge is

$$
\frac{Q_{R}}{e}=\frac{1}{e}\left[\int_{-e / 2}^{e / 2} Q^{2} \frac{d Q}{e}\right]^{1 / 2}=\frac{1}{\sqrt{12}},
$$




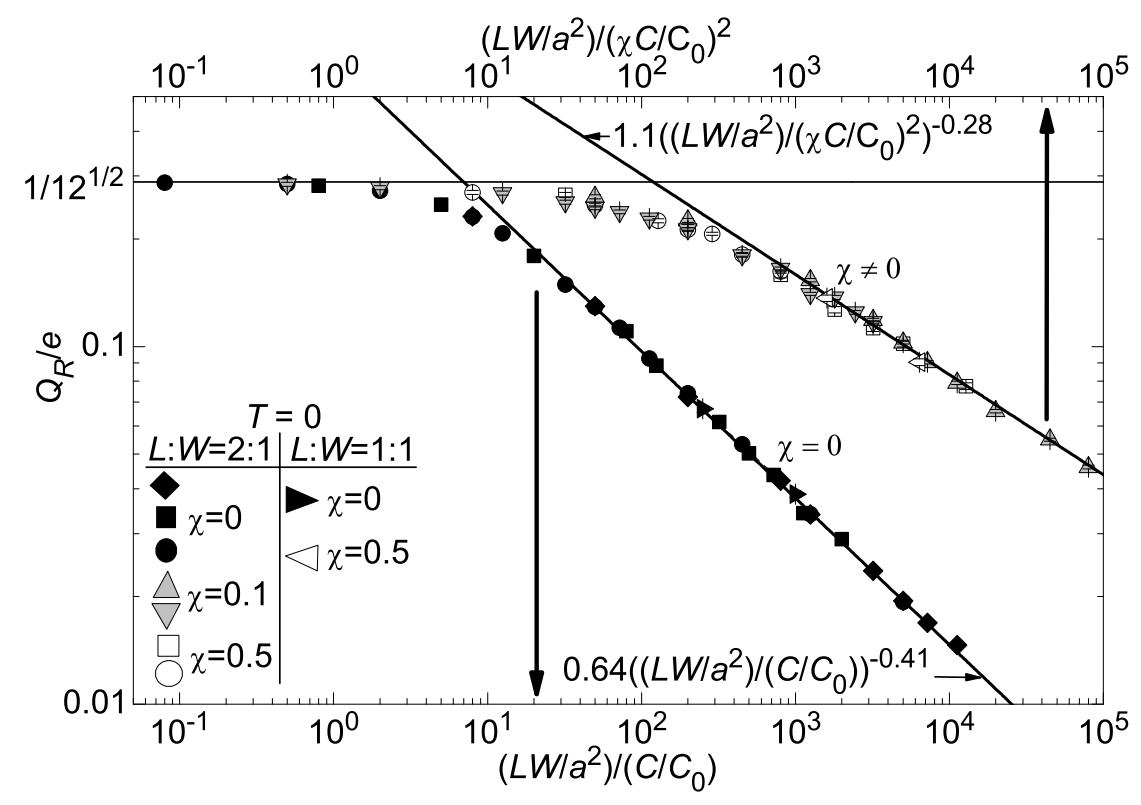

Figure 4. The same results for $Q_{R}$ as in Fig. 3, re-plotted to emphasize their universal scaling with system parameters. Solid lines show the best fits to the asymptotic behavior of $Q_{R}$ for large samples.

in a good accordance with the simulation results (Fig. 31).

On the other hand, if the conductor area is increased, $Q_{R}$ decreases, since there are more and more internal localized sites available for further charge relaxation. Our results (Fig. 31) show also that $Q_{R}$ always increases with capacitance $C$ and, at substantial Coulomb interaction, with its strength $\chi$.

Rather unexpectedly, we have found that for a broad range of system parameters, all these dependencies may be very well approximated by "universal" laws, different for the cases when Coulomb interaction is negligible $\left(\chi^{3} \ll Q_{R} / C L E_{0}\right)$ or substantial - see

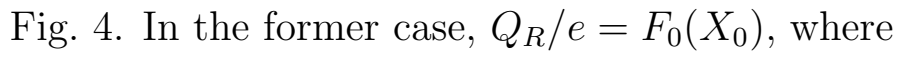

$$
X_{0}=\frac{L W}{a^{2}} \frac{C_{0}}{C}=L W \nu_{0} \frac{e^{2}}{C},
$$

while in the latter case $Q_{R} / e=F_{\chi}\left(X_{\chi}\right)$, where

$$
X_{\chi}=\frac{L W}{a^{2}} \frac{C_{0}^{2}}{\chi^{2} C^{2}}=\frac{L W \kappa^{2}}{C^{2}} .
$$

At small values of their arguments, both functions $F$ tend to $1 / \sqrt{12}$, in agreement with Eq. (9). Their asymptotic behavior is also functionally similar, $F(X) \rightarrow D X^{-\beta}$ at $X \rightarrow \infty$, but with different best-fit values of the coefficients: for $\chi=0, D=0.64 \pm 0.01$ and $\beta=0.41 \pm 0.01$, while for $\chi \sim 1, D=1.1 \pm 0.1$ and $\beta=0.28$, with the error bar about 0.03 for the dependence on $C$ and of the order of 0.01 for other variables contributing to $X_{\chi}$. 


\section{Discussion}

For the case of negligible Coulomb interaction, the asymptotic power law for function $F_{0}\left(X_{0}\right)$ may be readily explained, using the basic ideas of the Coulomb blockade [5]. Charge relaxation continues with the reduction of the system energy (on the average, dominated by the capacitor energy $U$ ) until the number $N$ of localized sites available for hopping becomes less than one. If the capacitance charge before a hop is $Q$, the range of capacitive energy of available initial sites is $\Delta U \sim Q^{2} / 2 C$, so that the average number of such sites per unit area is $n_{i} \sim \nu_{0} \Delta U \sim \nu_{0} Q^{2} / 2 C$, and their total number in the sample of area $L \times W$ is $N_{i} \sim L W n_{i} \sim L W \nu_{0} Q^{2} / 2 C$. In order to estimate $N$, we need to multiply $N_{i}$ by the average number $N_{f}$ of available final sites for each initial site. For small changes of charge, $|\Delta Q| \ll e$, the area $|\Delta x| \times W$ where such states can reside is much smaller than the sample area $L \times W$, because such charge change corresponds to a hop by distance $|\Delta x|=L \times|\Delta Q| / e \ll L$. Hence $N_{f} \sim L W \nu_{0}(|\Delta Q| / e)(Q-\Delta Q)^{2} / 2 C$ and we get the following estimate

$$
N \sim N_{i} N_{f} \sim\left(\frac{L W \nu_{0}}{2 C}\right)^{2} \frac{Q^{2}|\Delta Q|(Q-\Delta Q)^{2}}{e} .
$$

Now, from the natural requirement that $N$ drops below 1 as soon as $|Q|,|\Delta Q|$, and $|Q-\Delta Q|$ all become, on the average, of the order of $Q_{R}$, we get

$$
\frac{Q_{R}}{e} \sim\left(\frac{L W \nu_{0} e^{2}}{C}\right)^{-2 / 5}=X_{0}^{-2 / 5},
$$

which when compared to the power law $F(X)$ discussed above gives $\beta=2 / 5=0.40$, i.e. inside the narrow interval $0.41 \pm 0.01$ given by the numerical experiment.

For the case of substantial Coulomb interaction of hopping electrons, the situation is more complex - see, e.g., the discussion on pp. 435-443 of Ref. [3]. It is well documented that "external" transport (bringing electrons into and out of the hopping conductor) may be well understood in terms of the simple quasiparticles introduced by Efros and Shklovskii [2], with energy

$$
\varepsilon_{j} \equiv \varepsilon_{j}^{(0)}+\frac{e^{2}}{\kappa} \sum_{l \neq j}\left(n_{l}-\frac{1}{2}\right) G\left(\mathbf{r}_{j}, \mathbf{r}_{l}\right) .
$$

In $2 \mathrm{D}$ systems, their density of states at low energies $\epsilon$ is given by the famous Coulombgap expression [2]

$$
\nu(\varepsilon) \approx \frac{2 \kappa^{2}}{\pi e^{4}}|\varepsilon| .
$$

If we naively repeat the above calculation of $Q_{R}$, just replacing $\nu_{0}$ with $\nu(\epsilon)$ from the last expression, we get

$$
\frac{Q_{R}}{e} \sim\left(\frac{L W \kappa^{2}}{C^{2}}\right)^{-2 / 9}=X_{\chi}^{-2 / 9},
$$

i.e. the experimentally observed universality $\left(X_{\chi}=L W \kappa^{2} / C^{2}\right)$, but with an exponent $\beta=2 / 9 \approx 0.22$ which is significantly outside of the experimental interval $0.28 \pm 0.01$. 
Actually, for intra-sample transport, more adequate quasiparticles may be the so-called "dipole excitations" (essentially, electron-hole pairs with correlated energies) whose density $F(\Omega, r)$ depends on both the pair energy $\Omega$ and the distance $r$ between the pair components (see 3] p.435). In contrast to constant-field transport, the residual charge statistics are dominated by large-size pairs (hops), with $x$-component of the order of $L(|\Delta Q| / e)$ and $y$-component of the order of $W$. If we neglect, for such hops, the interaction of the pair components in comparison with $\Omega$, then $F$ depends only on energy:

$$
F(\Omega)=\int_{0}^{A} d \varepsilon_{1} \int_{-A}^{0} d \varepsilon_{2} \nu\left(\varepsilon_{1}\right) \nu\left(\varepsilon_{2}\right) \delta\left(\varepsilon_{1}-\varepsilon_{2}-\Omega\right) .
$$

For energies $\Omega$ much less than both the cutoff energy $A$ and the Coulomb gap width, this integral yields

$$
F=\left(\frac{2 \kappa^{2}}{\pi e^{4}}\right)^{2} \frac{\Omega^{3}}{6} .
$$

Now, following the arguments used above, we can accept $\Omega \sim Q^{2} / 2 C$, and take $L W$ for the possible area of the pair centers, and $L(|\Delta Q| / e) W$ for the pair area. After the integration of $F$ from 0 to $\Omega$, for the possible number of pairs within our energy range we get

$$
N \sim \frac{1}{24}\left(\frac{2 \kappa^{2}}{\pi e^{4}}\right)^{2}\left(\frac{Q^{2}}{2 C}\right)^{4} L^{2} W^{2} \frac{|\Delta Q|}{e} .
$$

Again, requiring that $N \sim 1$ at $Q,|\Delta Q| \sim Q_{R}$, we get back to the estimate given by Eq. (16).

It is not quite clear presently whether the discrepancy between these analytical arguments and the results of our numerical experiments may be overcome by an account of electron-hole pairs of smaller size, with strongly interacting pair components.

\section{Offset Charge Grounding}

The results of this work allow one to estimate the prospects of applying hopping conductors as "grounding" devices for the random background charge in single-electron devices. Figure 5 shows this idea on the example of a single-electron transistor [5. 9. Charged impurities, randomly located in the vicinity of the transistor's singleelectron island, induce on it a net polarization charge. The "integer" (e-multiple) part of this "background" charge is automatically compensated by tunneling through the transistor's tunnel junctions, but its fractional part $-e / 2<Q_{0}<+e / 2$ cannot be compensated in this way. This random charge is equivalent to a random shift $\Delta V_{g}=Q_{0} / C_{g}$ of the gate voltage; such shifts are one of the main obstacles on the way toward integrated circuits using single-electron devices, because for most of them the tolerable background charge range is as narrow as $\sim 0.1 e$. 9 . The problem may be solved by connecting the single-electron island to "ground" through a hopping conductor which would provide a slow relaxation of the background charge [9]. (For digital applications, 


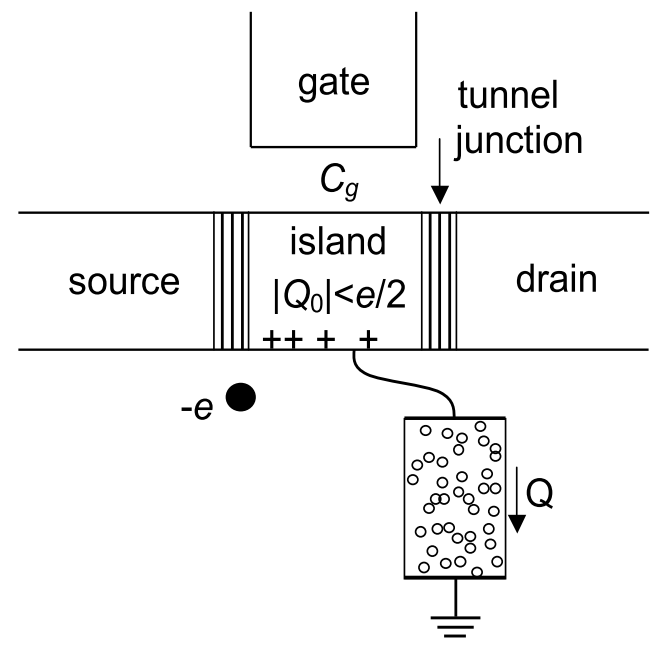

Figure 5. Background charge "grounding" using a hopping conductor (schematically).

the characteristic relaxation time has to be much longer than at least the circuit clock cycle, and more preferably the full time of the calculation performed by the circuit.)

For typical hopping conductors technologically compatible with silicon technology (e.g., amorphous semiconductors and metal oxides), the dielectric constant $\kappa$ is of the order of 10 , while the electron effective mass $m \sim 0.2 m_{0}$. This gives the localization radius $a \sim \hbar^{2} \kappa / m e^{2} \sim 3 \mathrm{~nm}$ and the level splitting scale $e^{2} / \kappa a \approx m e^{4} / \kappa^{2} \hbar^{2} \sim 30 \mathrm{meV}$. In order to stay on the dielectric side of the metal-insulator transition, the average distance between the localized sites should be above $\sim 4 a$ [1]; for the 3D density of states $\nu_{3}$ this gives the condition $\nu_{3} \lesssim 10^{19} \mathrm{eV}^{-1} \mathrm{~cm}^{-3}$. This condition is well satisfied, e.g., for most species of amorphous silicon, where $\nu_{3}$ at mid-bandgap is of the order of $10^{16} \mathrm{eV}^{-1} \mathrm{~cm}^{-3}$ (see, e.g., Ref. [32]). For thin films of such material with thickness $t \sim a \sim 3 \mathrm{~nm}$, the 2D density of states $\nu_{0} \sim 3 \times 10^{9} \mathrm{eV}^{-1} \mathrm{~cm}^{-2}$. For these parameters, the Coulomb interaction parameter $\chi$ is much smaller than 1, and we can use Eq. (13) for estimates. Even for the least demanding applications of single-electron devices, the electron addition energy $e^{2} / C$ should be at least $30 k_{B} T$ [9], so that according to Eq. (13), $X_{0}$ has to be above $\sim 300$.

Let us accept $L=W$ in order to minimize the conductors' self- ("stray") capacitance $C_{s}$ (which, as we will show shortly, may present a major problem) at fixed area $L \times W$. For the usual conditions of low-temperature experiments with singleelectron devices, $T \sim 0.1 \mathrm{~K}, C$ may be of the order of $10^{-14} \mathrm{~F}$, so that with our parameters $L$ should be above $\sim 30 \mathrm{~nm}$. This is less than the typical length $(\sim 1$ $\mu \mathrm{m}$ ) of the single-electron island in such experiments, so that the grounding idea may actually work 33 .

On the other hand, for the most important case of room-temperature single-electron devices $(T \approx 300 \mathrm{~K})$, the island capacitance should be much less, $C<10^{-18} \mathrm{~F}$, so that 
the quasi-continuous conduction is only possible at $L \gtrsim 15 \mu \mathrm{m}$. Stray capacitance $C_{s}$ of such a conductor would be larger than $\sim 10^{-15} \mathrm{~F}$, i.e. much larger than $C$, thus increasing the total effective capacitance of the island well above the acceptable value.

To summarize, our calculations indicate that the fractional charge grounding is possible, but practicable only for low-temperature experiments rather than for roomtemperature single-electron devices. Fortunately, by now an alternative way to solve (or rather circumvent) the random background charge problem in digital nanoelectronics has been suggested. This approach is based on reconfigurable hybrid CMOS-nanodevice digital circuits which may be re-routed around "bad" devices - see, e.g., Ref. [34]. Recent calculations have shown that this approach may provide defect tolerance up to $\sim 10 \%$ in memory circuits and $>20 \%$ in logic circuits. This is much higher than the estimated lower bound on the fraction $(\sim 0.1 \%[9])$ of single-electron devices whose threshold is substantially shifted by random background charges.

\section{Acknowledgments}

The authors would like to thank B. I. Shklovskii for numerous illuminating discussions. Useful comments by A. Efros, T. Grenet, A. N. Korotkov, A. Möbius, M. Pollak and V. A. Sverdlov are also gratefully acknowledged. The work was supported in part by the Engineering Physics Program of the Office of Basic Energy Sciences at the U.S. Department of Energy, and by the Semiconductor Research Corporation. We also acknowledge the use of the following supercomputer resources: our group's cluster $N j a l$ (purchase and installation funded by U.S. DoD's DURINT program via AFOSR), Oak Ridge National Laboratory's IBM SP computer Eagle (funded by the Department of Energy's Office of Science and Energy Efficiency program), and also IBM SP system Tempest at Maui High Performance Computing Center and IBM SP system Habu at NAVO Shared Resource Center (computer time granted by DOD's High Performance Computing Modernization Program).

\section{References}

[1] N. F. Mott and J. H. Davies, Electronic Properties of Non-Crystalline Materials, 2nd Ed. (Oxford Univ. Press, Oxford, 1979); N. F. Mott, Conduction in Non-Crystalline Materials, 2nd Ed. (Clarendon Press, Oxford, 1993).

[2] B. I. Shklovskii and A. L. Efros, Electronic Properties of Doped Semiconductors (Springer, Berlin, 1984).

[3] A. L. Efros and B. I. Shklovskii, "Coulomb Interaction in Systems with Localized States", in Hopping Transport in Solids, edited by M. Pollak and B. Shklovskii (North-Holland, Amsterdam, 1991).

[4] Sh. Kogan, Electronic Noise and Fluctuations in Solids (Cambridge University Press, Cambridge, 1996).

[5] D. V. Averin and K. K. Likharev, "Single-Electronics", in Mesoscopic Phenomena in Solids, edited by B. Altshuler et al. (Elsevier, Amsterdam, 1991), pp. 173-271; see specifically p. 257.

[6] K. A. Matsuoka and K. K. Likharev, Phys. Rev. B 57, 15613 (1998). 
[7] D. Kaplan, Y. Kinkhabwala, A. Korotkov, V. Sverdlov, and K. Likharev, "Sub-electron Charge Transport in Nanostructures", in Proc. of the 20th Symposium on Energy Engineering Sciences, ANL, Agronne, IL, 2002), pp. 231-240.

[8] Y. Naveh, D. Averin, and K. Likharev, Phys. Rev. B 58, 15371 (1998).

[9] K. K. Likharev, Proc. of IEEE 87, 606 (1999).

[10] M. J. M. de Jong and C. W. J. Beenakker, "Shot Noise in Mesoscopic Systems", in Mesoscopic Electron Transport, edited by L. L. Sohn, L. P. Kouwenhoven, and G. Schön, NATO ASI Series Vol. 345 (Kluwer Academic Publishers, Dordrecht, 1997), p.225.

[11] Ya. M. Blanter and M. Buttiker, Phys. Repts. 336, 2 (2000).

[12] V. V. Kuznetsov, E. E. Mendez, X. Zuo, G. Snider, and E. Croke, Phys. Rev. Lett. 85, 397 (2000).

[13] S. H. Roshko, S. S. Safonov, A. K. Savchenko, W. R. Tribe, and E. H. Linfield, Physica E 12, 861 (2002).

[14] A. N. Korotkov and K. K. Likharev, Phys. Rev. B 61, 15975 (2000).

[15] V. A. Sverdlov, A. N. Korotkov, and K. K. Likharev, Phys. Rev. B 63, 081302(R) (2001).

[16] Y. A. Kinkhabwala, V. A. Sverdlov, A. N. Korotkov, and K. K. Likharev, J. Phys.: Condens. Matter 18, 1999 (2006).

[17] Y. A. Kinkhabwala, V. A. Sverdlov and K. K. Likharev, J. Phys.: Condens. Matter. 18, 2013 (2006).

[18] J. Lambe and R. C. Jaklevic, Phys. Rev. Lett. 22, 1371 (1969).

[19] L. S. Kuzmin and K. K. Likharev, JETP Lett. 45, 495 (1987).

[20] Following most studies of the Coulomb interaction at hopping, we keep the conductor electroneutral by adding an effective background charge of $-e / 2$ to each localized site.

[21] A. B. Bortz, M. H. Kalos, and J. L. Leibowitz, J. Comp. Phys. 17, 10 (1975).

[22] N. S. Bakhvalov, G. S. Kazacha, K. K. Likharev, and S. I. Serdyukova, Sov. Phys. JETP 68, 581 (1989).

[23] C. Wasshuber, Computational Single-Electronics (Springer, Berlin, 2001), Ch. 3.

[24] C. J. Adkins, J. D. Benjamin, J. M. D. Thomas, J. W. Gardner, and A. J. McCeown, J. Phys. C 17, 4633 (1984).

[25] Z. Ovadyahu and M. Pollak, Phys. Rev. B 68, 184204 (2003).

[26] T. Grenet, Eur. Phys. J. B 32, 275 (2003); Phys. Stat. Sol. (c) 1, 9 (2004).

[27] B. I. Shklovskii, Sov. Phys. Semicond. 6, 1964 (1973).

[28] N. Apsley and H. P. Hughes, Philos. Mag. 30, 963 (1974); 31, 1327 (1975).

[29] M. Pollack and I. Riess, J. Phys. C 9, 2339 (1976).

[30] R. Rentzsch, I. S. Shlimak and H. Berger, Phys. Status Solidi A 54, 487 (1979).

[31] M. van der Meer, R. Schuchardt and R. Keiper, Phys. Status Solidi B 110, 571 (1982).

[32] T. Sameshita and S. Usui, J. Appl. Phys. 70, 1281 (1991).

[33] Actually, the first qualitative observations of relaxation of sub-electron background charge to $Q_{R} \ll e$ in early experiments [18, 19] may be considered as the first, albeit unintentional implementations of this idea.

[34] K. K. Likharev and D. V. Strukov, "CMOL: Devices, Circuits, and Architectures", in Introducing Molecular Electronics, edited by G. Cuniberti et al. (Springer, Berlin, 2005), pp. 447-477. 